\title{
Los Actores Políticos y Sociales en la Formulación de la Reforma Sanitaria, en España ${ }^{1}$
}

\section{Social and Political Actors in the Formulation of Health Reform in Spain}

\author{
Joaquina E. Etviti ${ }^{2}$
}

René F. Leyva ${ }^{2}$

\section{ERVITl, J. E. \& LEYVA, R F. Social and Political Actors in the Formulation of Health Reform in} Spain. Cad. Saúde Públ., Rio de Janeiro, 11 (1): 34-44, Jan/Mar, 1995.

An analysis of the participation of social and political actors in the process of formulating health policy allows one to understand the specific characteristics of the organization and operation of a health system.

This study analyzes the drafting process for the General Health Act $(L G S)$ in Spain with the purpose of establishing the relationship between social, political, and economic actors in both the formulation of the Act itself and the organization of the Spanish Health System. A case study was carried out from 1982 through 1986. Documentary parliamentary data, the medical press, national magazines and journals, and press reports by political, social, and public health actors were analyzed.

The first version of the General Health Act presented by the Spanish Socialist Workers' Party (PSOE) proposed a health system with funding and public administration aimed at achieving universal health coverage, integrated care, community participation, and health education. This proposal was submitted to a complex negotiating process with business groups, unions, and health professionals. The General Health Act as finally approved excludes the principles of equity and incorporates private interests in health: "free choice of doctor and hospital", public funding and private administration of the health system, and the establishment of Social Security as the core of the entire health system.

Key words: Policy; Health Systems; Social Actors; Health Coverage; Social Security

\section{INTRODUCCION}

\section{La Transición Política}

La formulación de la propuesta de reforma sanitaria en España se da en el marco de un proceso de transición política de dictadura hacia democracia. El proceso se inició en un régimen autoritario en crisis que se caracterizó por reservar "lo político" al Jefe de Estado como

\footnotetext{
${ }^{1}$ Ponencia presentada en el $6^{\circ}$ Congreso Latinoamericano y $8^{\circ}$ Congreso Mundial de Medicina Social en Guadalajara, Jalisco, del 20 al 23 de marzo de 1994.

${ }^{2}$ Instituto Nacional de Salud Pública, Escuela de Salud Pública de México. Av. Universidad 655, Col. Sta María Ahuacatitlan, C.P. 62508, Cuernavaca, Morelos México.
}

representante de los intereses de los españoles (Del Aguila \& Montoro, 1984).

La transición política giró alrededor del término consenso, asociado a lo largo del tiempo con reconciliación, convivencia, moderación, tolerancia, acuerdo y pacto racional, corno mecanismo que ayudara a salvar la "débil" democracia. Esto, si bien ayudó al asentamiento de la democracia, lo hizo con importantes concesiones desde la izquierda (García, 1987). Pactos y acuerdos se ofrecían como resultado de un proceso al que se asistía como mero espectador. La discusión pública desaparecía y reaparecía en cadencias observadas con cierto estupor y las decisiones siguieron tomándose tras el fondo del escenario y no sobre él, en to privado de la esfera y no en su vertiente pública (Del Aguila \& Montoro, 1984). 
Estos años estuvieron presididos por una gran crisis económica con estancamiento económico, disminución de la inversión en capital fijo, inflación y desempleo. La inflación no bajó del $15 \%$ en todo el período, con un punto álgido de $29 \%$ en 1977. El desempleo presentó un crecimiento continuo y dramático, desde $2.8 \%$ en 1973 hasta aproximadamente el $22 \%$ en 1987, con tres millones de parados y una cobertura de seguro de desempleo del 27\%; el desempleo juvenil alcanzó casi el 50\% (Maravall, 1981; Ortún, 1986).

El movimiento obrero mantuvo una importante presión en los primeros años de la transición. Esta fue reduciéndose con las Ilamadas a desmovilizaciones y por la reforma pactada desde la izquierda. En 1978 se redujo el número de huelgas casi a la mitad y la mayoría fueron por reivindicaciones socioeconómices (Maravall, 1981).

\section{El Debate de la Reforma Sanitaria en España}

En el área de la salud se reclamaba una reforma sanitaria para el país, especialmente por los médicos jóvenes. Estas demandas fueron incorporadas, especialmente por los partidos de izquierda, y debatidas en el Parlamento. A pesar de ello, la reforma sanitaria estaba pendiente y fue una de las promesas electorales del Partido Socialista
Obrero Español (PSOE), en su campaña "por el cambio" en 1982. Para ello, se formuló (1983) y aprobó (1986) la Ley General de Sanidad (LGS) como instrumento legal para la reforma sanitaria. Sin embargo, lo que en un principio se prometía como un trámite rápido y pacífico, se convirtió en uno de los aspectos más polémicos de la política social, provocando grandes movilizaciones, principalmente por los médicos, liderados por la Organización Médica Colegial (OMC) que se oponía a la reforma sanitaria propuesta por el Gobierno-PSOE.

El proceso de formulación se inició con el nombramiento de la Comisión redactora del Proyecto en 1983, a un mes de formado el primer gobierno del PSOE, y tardó un año en presentar públicamente el primer Borrador (Comisión Redactora, 1984) de la LGS que fue objeto de negociaciones privadas durante 1984. El Consejo de Ministros envió el Anteproyecto (Gobierno Español-Consejo de Ministros, 1985) de LGS al Congreso de los Diputados para su discusión pública en 1985. Tras un año de discusión y aprobación o rechazo de enmiendas en el Parlamento y en el Senado, la Ley (Gobierno Español-Congreso de Diputados, 1986) se aprobó en abril de 1986, días antes de la disolución de las Cortes para elecciones generales anticipadas. El proceso de formulación, hasta su publicación como Ley, abarcó el primer período de gobierno del PSOE, 1982-1986 (Tabla 1).

TABLA 1. Proceso de Formulación de la Ley General de Sanidad

\begin{tabular}{|l|l|l|l|l|}
\hline \hline Periodo & \multicolumn{1}{|c|}{$1982-83$} & \multicolumn{1}{|c|}{1984} & \multicolumn{1}{c|}{1985} & \multicolumn{1}{c|}{1986} \\
\hline \hline Proceso & $\begin{array}{l}\text { Elaboración del } \\
\text { Anteproyecto }\end{array}$ & $\begin{array}{l}\text { Discusión del } \\
\text { Anteproyecto }\end{array}$ & $\begin{array}{l}\text { Discusión del } \\
\text { Proyecto de Ley }\end{array}$ & $\begin{array}{l}\text { Publicación de la } \\
\text { LGS }\end{array}$ \\
\hline Actores & $\begin{array}{l}\text { Comissión } \\
\text { Redactora }\end{array}$ & $\begin{array}{l}\text { Gobierno, OMC, } \\
\text { CEOE, FADSP }\end{array}$ & $\begin{array}{l}\text { Partidos políticas y } \\
\text { otros actores. }\end{array}$ & \\
\hline Espacios de & Esfera privada. & $\begin{array}{l}\text { Esfera privada: } \\
\text { Ministerio de } \\
\text { Sanidad y actores }\end{array}$ & $\begin{array}{l}\text { Esfera pública, } \\
\text { Parlamento y } \\
\text { yenado, }\end{array}$ & \\
\hline \hline
\end{tabular}


Durante el período de formulación de la Ley se inició la implantación de la reforma sanitaria, a través de una normativa menor, que abarcó aspectos de la reforma hospitalaria y extrahospitalaria (creación de Unidades Básicas de Salud), y de reglamentos que incidían en el control de la gestión de recursos humanos y materiales, relativos al cumplimiento de horario, las convocatorias para contratación del personal sanitario, incompatibilidades de horarios e instituciones, control de recetas, entre otros, afectando intereses profesionales de los médicos.

La presente investigación analizó el proceso de formulación de la LGS a partir de la propuesta gubernamental y de la posición de los distintos actores políticos, sociales y económicos para conocer cuál fue el impacto de su participación en la LGS y en la conformación del sistema de salud español.

\section{METODOLOGIA}

Se realizó un estudio de caso sobre el proceso de formulación de la LGS en España que comprendió el período 1982-1986. Para ello, se analizó la participación de los actores políticos, sociales y sanitarios, su discurso, sus acciones y sus propuestas para la formulación de la LGS, instrumento legal para la reglamentación, organización y funcionamiento del Sistema Nacional de Salud.

La sistematización del proceso de formulación se realizó a través de una revisión documental tortuosa, que en parte es expresión ya histórica de la ausencia de una cultura democrática en España, que diferencia claramente lo público de lo privado en los procesos políticos.

Se utilizó la documentación parlamentaria referente al problema como expresión de la participación en la esfera pública, y en el intento de extraer la participación en la esfera privada, se hizo una revisión de prensa médica, principales diarios y revistas a nivel nacional, así como documentación, informes y prensa de los respectivos actores politicos, sociales y sanitarios, referida al tema. En estos documentos se identificaron las propuestas organizativas, financieras, y de gestión para la conformación del modelo sanitario.

Se tomó como base para realizar un análisis comparativo el primer Borrador de la LGS presentado por el Gobierno en 1984, el Proyecto de Ley enviado por el Gobierno al Congreso de los Diputados y la LGS aprobada por el Congreso y publicada como Ley.

Los actores fueron caracterizados en tres grandes grupos: actores políticos, actores sanitarios y organizaciones empresariales y sindicales (Tabla 2).

TABLA 2. Actores Políticos y Sociales

\begin{tabular}{|c|c|c|c|}
\hline \hline \multirow{2}{*}{ A. Políticos } & \multicolumn{3}{|c|}{ Actores Sociales } \\
\hline \hline \multirow{2}{*}{$\begin{array}{c}\text { PSOE } \\
\text { (socialistas) }\end{array}$} & Económicos & Sindicales & Sanitarios \\
\cline { 2 - 4 } & org. empresarios & UGT & OMC \\
(socialista) & (col. médicos) \\
\hline PCE & IF & CCOO & FADSP \\
(comunistas) & i. farmacéutica & aso. s. pública \\
\hline AP & & & Colegios de \\
(conservador) & & Farmacéuticos \\
\hline PNV, CiU & & CESM, FESIME \\
(regionales) & & (sind. médicos) \\
\hline \hline
\end{tabular}


Los actores políticos, con representación parlamentaria a nivel nacional o regional, con mayor protagonismo en el debate de la LGS fueron el Partido Socialista Obrero Español (PSOE), con mayoría parlamentaria, el Partido Comunista (PCE), el partido de derecha Alianza Popular (AP) Y los partidos regionalistas Partido Nacionalista Vasco (PNV), en el País Vasco, y Convergencia i Unió (CiU), en Cataluña.

Los actores sanitarios que participaron activamente en el proceso fueron los Colegios de Médicos (OMC) y de Farmacéuticos; los sindicatos médicos: Confederación Española de Sindicatos Médicos (CESM) y Federación de Sindicatos Médicos (FESIME); y la Federación de Asociaciones para la Defense de la Sanidad Pública (FADSP).

En las Organizaciones Empresariales, la Confederación Española de Organizaciones Empresariales (CEDE) y, en el rubro de Industria Médico-Fatmacéutica (IF), se integraron las representaciones empresariales del área. Las Organizaciones Sindicales más representativas a nivel nacional que patticiparon fueron Unión General de Trabajadores (UGT), sindicato socialista, y Comisiones Obreras (CCOO), sindicato comunista.

Otras organizaciones como las Asociaciones de Usuarios, recién creadas, y las Asociaciones de Vecinos, que tuvieron su auge en la década de los setenta, con vocalías de higiene y sanidad, aunque son incluidas en el discurso oficial, no tuvieron participación en el proceso de elaboración de la LGS.

\section{RESULTADOS}

\section{La Propuesta de Reforma Sanitaria}

El primer Borrador de la LGS, presentado por el Gobiemo, reflejaba en gran medida la promesa electoral del PSOE en el aspecto sanitario. Se señalaban como principios inspiradores del anteproyecto: la extensión de cobertura sanitaria a toda la población, un concepto integral de salud, la participación comunitaria y la educación sanitaria. Se proponía la creación de un Servicio Nacional de Salud financiado por fondos públicos, gestionado pot los poderes públicos y cuyas prestaciones alcanzaran a toda la población (Mancilla, 1984).

Este Servicio Nacional de Salud estaría constituido por los servicios de salud de las diecisiete Comunidades Autónomas y pot el Servicio Central de Salud. Para elio, se requería a las Comunidades Autónomas iniciar un proceso de integración de las diversas instituciones sanitarias como base pare la conformación de este Servicio Nacional de Salud. Se definieron las Areas de Salud, entendidas como los espacios donde deben existir recursos suficientes para atender a la población situada en su circunscripción. Asimismo se propuso un Consejo de Dirección, con mayoría de la Comunidad Autónoma, 60\%, y una representación de los municipios con un 40\% (Mancilla, 1984).

En definitiva, el modelo que se proponía era un Servicio Nacional de Salud, universal, con financiamiento público y gestión pública de los recursos, planificación central y local, con libre elección de médico al interior de un equipo de salud y limitación de conciertos con la medicina privada.

\section{La OMC y el Anteproyecto de LGS}

La OMC, con el propósito de presentarse como un organismo aglutinador de los intereses politicos, administrativos y económicos que pudieran set afectados por la LGS, criticó el Borrador en sus aspectos juridicos, técnico-sanitarios y deontológicos (CGCOF, 1984). Identificó como bloque opositor a Ia LGS a las Comunidades Autónomas y municipios, la industria farmacéutica, las clínicas privadas, los médicos y los usuarios.

Reclamaban la inconstitucionalidad del Anteproyecto en relación a la interferencia en las competencies de las Comunidades Autónomas y en la autonomía local de los municipios para establecer modelos sanitarios privados o mixtos, más acordes con los intereses representados en la OMC.

Calificaron al modelo sanitario como "estatalizador y centralists", limitante de "la iniciativa privada", reclamando el principio de 
libre empresa y el apoyo de los poderes públicos en la creación de condiciones de demanda y consumo. Esto traducido en financiamiento público y gestión privada de la salud pública y las prestaciones sanitarias (OMC, 1985).

Desde su valoración "técnico-sanitaria" presentan como culpable de la mala situación de la sanidad en España a la red de Asistencia Sanitaria de la Seguridad Social. La propuesta de la OMC resalta la más amplia libertad de elección de médico y centro hospitalario, y la competencia entre éstos como garantía "para alcanzar eficacia y calidad asistencial" (OMC, 1985). Su esquema de operacionalización fue la utilización de los centros privados dentro del sistema sanitario que contribuiría, además, a reducir los costos de atención. Estas afirmaciones no fueron apoyadas pot estudios respectivos. Se autodefinieron como los poseedores de los conocimientos técnicos para la dirección y control del sistema sanitario.

La salud integral, uno de los objetivos del Proyecto del Gobierno, que contemplaba la atención preventiva y curativa por un equipo multidisciplinário, fue considerada como "exclusivamente aplicable a sociedades tercermundistas". Por el contrario, proponían una sanidad integral referida a la coordinación entre niveles de atención, con equipos para actividades preventivas diferentes a los asistenciales.

En resumen, la propuesta de la OMC fue conformar un sistema de atención privado o mixto con financiamiento público y gestión privada de los recursos, coordinación de instituciones versus integración, con la más amplia libertad de elección de médico y centro hospitalario y la no restricción de convenios del sistema público con el privado.

Los Colegios de Farrnacéuticos buscaron introducir modificaciones en el capítulo referido a productos farmacéuticos con el propósito de continuar como los únicos proveedores de medicamentos a la Seguridad Social, salvo el consumo intrahospitalario de medicamentos. Junto con la Industria Farmacéutica se declararon contrarios al incremento de los porcentajes de pago por el usuario y a la restricción de insumos en el Sistema Sanitario como factor disuasorio del consumo (Sánchez, 1985).

\section{La FADSP y el Anteproyecto}

La FADSP y otras agrupaciones como CCOO, UGT, PCE y Asociaciones de Vecinos son actores cuya propuesta de modelo sanitario coincidía, en gran parte, con el Borrador de LGS. Su propuesta, un Servicio Nacional de Salud, universal, financiado con presupuestos de las administraciones públicas a sus diferentes niveles, por la vía de la fiscalidad (FADSP, s/f).

Criticaron el Anteproyecto enviado al Congreso, generado despues de un año de negociaciones, por presentar de manera contradictoria la relación entre la cobertura universal de atención a la salud y el financiamiento del sistema. Es decir, se habla de derecho a la salud de "todos los españoles" y se establece como titulares reales del derecho a los asegurados de la Seguridad Social, los ciudadanos que puedan acreditar no tener "recursos económicos", y los que paguen por "proceso sanitario" o por "pago periódico". Sin embargo, el Ministerio de Sanidad argumentó que era reducido el número $\left(3^{\circ} . \mathrm{b}\right)$ de ciudadanos sin cobertura.

Por su parte, la FADSP enfatizó lo insolidario y humillante que representa para un grupo de ciudadanos el hecho de tener que probar su carencia de recursos pats poder tenet derecho a las prestaciones sanitarias. Estos por sus características de marginación socioeconómica presentan mayores necesidades de atención y tienen especiales dificultades para acudir a las prestaciones sanitarias. Consideraron que el Anteproyecto perfila un nuevo sistema sanitario mixto que garantiza amplias cuotas de mercado al sector privado, tanto a nivel de centros de atención, como de contratos, industria farmacéutica y tecnología (Sánchez, 1985), reconociéndose la vinculación de centros privados al sistema público (FADSP, s/f).

La FADSP propuso que la LGS debiera delimitar claramente las competencias del sector público y privado, señalando que los conciertos con entidades privadas sólo deberán realiza-se en caso de imposibilidad absoluta del Sistema Nacional de Salud para asumir unos servicios concretas y al mismo 
tiempo establecer plazos para la consecución la cobertura universal.

Su posición es contraria a la libre elección de médico porque favorece mecanismos de aumento de gasto, como medicina de complacencia, incremento de extensión de bajas, imposibilidad de control en el diagnóstico y terapéutica. Además dificulta la programación y planificación de recursos en base a las necesidades sanitarias. Consideran que la libre elección de médico no es un bien en si mismo, sino concebido como un derecho de los usuarios dentro de la planificación sanitaria global y al interior de equipos de atención primaria (FADSP, s/f) (Tabla 3).

Por otra parte, la FADSP estimó que la participación social en la gestión de salud era prácticamente inexistente, debido a la ausencia de estructuras de gestión.

\section{La CEOE y el Anteproyecto}

Desde la CEOE se criticó el sistema de financiamiento propuesto en el Borrador. Planteó una posición clara contra la expansión del sector público y contra todo lo que pudiera suponer un mayor control, contra la "confiscación del patrimonio de la Seguridad Social" que ignora a empresarios y trabajadores, quienes han contribuido a su formación, aunque "con una aportación sensiblemente superior de los primeros" (CEOE, 1984: 05).

Esta organización criticó la propuesta de participación en el Borrador, señalando que se debe priorizar la participación de los intereses de la Seguridad Social, las Organizaciones Empresariales, frente a los municipios y las Comunidades Autónomas.

Como la OMC, criticaron la propuesta de modelo sanitario público por inconstitucional, ineficiente y antisocial, reclamando como inaceptable el control público sobre la apertura de centros privados, inspecciones, procedimientos de control y evaluación, calificándolo como "intromisiones intolerables en la gestión interna de las empresas” (CEOE, 1984: 07) y exigieron la más amplia libertad de elección de médico y centro hospitalario.

Para la CEOE es "confuso" el capítulo sobre salud laboral pues "no alcanzamos a comprender qué signicado tenga la indicación de extender la prevención de los riesgos del medio ambiente laboral a la familia y a la comunidad" (CEOE, 1984: 05). Asimismo, están contra la participación de los sindicatos en la salud ocupacional, que es función, dicen, de los Comités de Seguridad e Higiene en el Trabajo (trabajadores y empresarios).

\section{Comunidades Autónomas}

Las demandas se referían, principalmente, a modificaciones en todos los aspectos referentes a merma de sus competencias por parte del Estado. Su objetivo era lograr la menor concreción en la Ley que permitiera el desarrollo posterior en forma autónoma (no uniforme) por cada Comunidad. Es importante señalar que ciertas concesiones del gobierno a las Comunidades Autónomas, pilares del Sistema Nacional de Salud, podían servirle para mostrar cierto consenso en la Ley y facilitar su desarrollo.

\section{Modificaciones en la LGS}

Las modificaciones más importantes en relación al financiamiento, a la organización, y a la planificación del sistema sanitario que aparecen en la LGS se resumen en la Tabla 4.

\section{Financiamiento}

El Borrador establecía como fuente de financiamiento a los presupuestos de las administraciones públicas, con aportaciones de las Comunidades Autónomas, Diputaciones, Ayuntamientos y otros entes territoriales competentes en materia de sanidad. En la LGS se establecieron tres vías para el sistema de financiamiento: los recursos de las administraciones públicas, las cotizaciones a la Seguridad Social y las tasas por prestación de servicios. Con ello, el tema de la gratuidad y la igualdad de acceso a los servicios de salud quedó regulado para determinados grupos de población. El Estado se encargará de contribuir financieramente al sostenimiento de la Seguridad Social y de compensarla por la extensión de la Asistencia Sanitaria a aquéllas personas sin 
recursos económicos. En opinión dal PSOE estos cambios "no han sido en los principios sino en las estrategias". Señalan que, en la medida de las posibilidades presupuestarias, irá creciendo la aportación Estatal y disminuirá la procedente de la Seguridad Social. "El partido tuvo que reconsiderar sus posiciones" (Fernández, 1986: 52).

TABLA 3. Propuestas de los Actores Político-Sociales para la Formulación de la Ley General de Sanidad

\begin{tabular}{|c|c|c|c|c|}
\hline Sistema Sanitario & PSOE (Borrador) & $\mathrm{OMC}$ & CEDE & FADSP \\
\hline Modelo Sanitario & público y universal & mixto o privado & seguridad social & público y universal \\
\hline Financiamiento & $\begin{array}{l}\text { fondos públicos y } \\
\text { gestión pública }\end{array}$ & $\begin{array}{l}\text { gestión privada de } \\
\text { los recursos }\end{array}$ & $\begin{array}{l}\text { Estado, empresarios } \\
\text { y trabajadores }\end{array}$ & $\begin{array}{l}\text { fondos públicos } \\
\text { gestión pública }\end{array}$ \\
\hline Planificación: & - integración & - coordinación & & •integración \\
\hline $\begin{array}{l}\text { a) Libre elección de } \\
\text { médico y hospital }\end{array}$ & - restringida & • amplia & - amplia & - restringida \\
\hline $\begin{array}{l}\text { b) Conciertos Libre } \\
\text { empresa }\end{array}$ & - restringidos & - amplio & - arnplio & - restringidos \\
\hline
\end{tabular}

TABLA 4. Modificaciones a la Propuesta de Ley General de Sanidad

\begin{tabular}{|l|l|l|}
\hline \hline \multicolumn{1}{|c|}{ Variable } & \multicolumn{1}{|c|}{ Borrador } & \multicolumn{1}{|c|}{ LGS } \\
\hline \hline Financiamiento & $\begin{array}{l}\text { Fondos públicos, vía impuestos, gestión } \\
\text { pública }\end{array}$ & $\begin{array}{l}\text { Estado, empresarios y trabajadores (cuotas a } \\
\text { la Seguridad Social) y pago } \\
\text { por servicio }\end{array}$ \\
\hline Organización & $\begin{array}{l}\text { Servicio Nacional de Salud, universal y } \\
\text { gratuito }\end{array}$ & Sistema Nacional de Salud \\
\hline Planificación & $\begin{array}{l}\text { Integración de redes sanitárias. } \\
\text { restringida al interior de un equipo } \\
\text { - Restricción de conciertos con el } \\
\text { sistema privado }\end{array}$ & $\begin{array}{l}\text { Coordinación de redes sanitarias. } \\
\text { hospital. } \\
\text { - Apertura para conciertos del sistema } \\
\text { público con el privado. } \\
\bullet \text { Vinculación del sistema privado al público }\end{array}$ \\
\hline \hline
\end{tabular}

\section{Organización}

La propuesta de conformar un Servicio Nacional de Salud fue substituida por la de Sistema Nacional de Salud. El PSOE argumentó "un obstáculo constitucional" para utilizar el término Servicio Nacional en un Estado-Nación con regiones autonómicas, y que el cambio de nombre sólo representaba una traducción jurídica (De Vicente, 1985).

Sin embargo, este cambio significó la introducción de la Seguridad Social, en el texto de la LGS, como rector del Sistema Nacional de
Salud. La Seguridad Social no era mencionada, en el Borrador de la LGS, en opinión de la OMC por las "dificultades técnicas y políticas qua entraña una expropiación" (OMC, 1985: 09), en tanto los centros y establecimientos de la Seguridad Social no son de "titularidad estatal” par ser ésta un organismo autónomo.

El establecemiento de áreas de salud e integración de la red sanitaria única, la atención integral a la salud y la participación de los gobiernos locales en la gestión pasó a un segundo plano de la organización. La propuesta de integración de redes sanitarias se sustituyó 
por la de coordinación. Para ésto se creó el Consejo Interterritorial del Sistema Nacional de Salud y un Comité Consultivo vinculado a él, integrado paritariamente por las organizaciones sindicales y empresariales más representativas.

En los Consejos de Salud de las Areas, considerados órganos colegiados de participación comunitaria para la consulta y el seguimiento de la gestión, no se reconoció la representación del personal de salud "no profesional". En su lugar se integraron, las corporaciones locales con un 50\%, las organizaciones sindicales, a través de los profesionales sanitarios titulados, en una proporción no inferior al 25\%, y el resto sería la administración sanitaria del área de salud.

\section{Planificación}

Los aspectos que se recogieron en la LGS y que modificaron y afectaron el proceso de planificación fueron: el más amplio reconocimiento de la libertad de elección de médico y centro hospitalario, la posibilidad de vinculación de centros privados a la red de centros públicos, y una menor regulación pare los conciertos de la red pública con centros privados.

La libre elección de médico se reconoció en el conjunto de la ciudad para poblaciones mayores de 250 mil. Los servicios privados se articulan al sistema público a través del reconocimiento al derecho "de libre empresa en el sector sanitario", desapareciendo "el principio de subsidiariedad del sistema privado con respecto al público" que se reeonocía en el Borrador. También, se reduce la regulación en la aperture de centros privados. Por otra parte, se establece la imposibilidad de vinculación al Sistema Nacional de Salud de los centros privados cuando en alguno de sus propietarios o trabajadores pueda darse la incompatibilidad de la función pública y privada.

\section{DISCUSIONY CONCLUSIONES}

La formulación de la LGS se da en un marco de reformas, desde 1977, con la elaboración de una nueva Constitución, impulsadas por el gobierno, resultado de un proceso consensual donde se expresaron los intereses sociales, económicos y politicos en torno a la salud de los españoles. El consenso permitió la elaboración de una Constitución y de la LGS, entre otras, "evitando enfrentamientos, conflictos y traumas"; sin embargo los costes no fueron menos importantes, la apatía y desmovilización en grandes sectores de población. La discusión pública desapareció y las decisiones se tomaron tras el fondo del escenario (Maravall, 1981; Del Aguila \& Montoro, 1984).

¿Cuáles fueron las consecuencias de la LGS en la conformación del nuevo sistema sanitario español? ¿Cómo afectaron a la población las nuevas regulaciones establecidas por la LGS? ¿Cuáles fueron los principales grupos de interés beneficiados con las reformas de la LGS?

Las variables que se tomaron en cuenta pare el análisis permitieron identificar cambios sustanciales entre la propuesta inicial plasmada en el Borrador y lo expresado en la LGS. Estos cambios presentan relación directa con los grupos de interés que participaron en el proceso de formulación de la LGS.

Esencialmente, la propuesta inicial planteaba un sistema sanitario público, universal, gratuito, con una concepción integral de salud y un sistema integrado por redes sanitarias. Los cambios expresados en la LGS se muestran, por una parte, como concesiones necesarias en busca de consenso y, por otra, como la adecuación al modelo económico-politico orientado hacia la privatización de los servicios sociales que asumía el Estado. Este modelo mixto, público-privado, con la exclusión de gran parte de lo que podía significar control desde el Estado, favoreció el desarrollo de estrategias de privatización, "liberalización" según el Gobierno. Entre las estrategias que los intereses privados defendieron para el "bienestar público" se encuentran la fibre elección de médico y centro hospitalario, la reducción de los porcentajes de pago por medicamento a los usuarios, y la oferta ilimitada de productos médicos. Estos aspectos tuvieron un amplio respaldo y consenso en la población usuaria, no 
obstante que, por sí mismos, no aseguran ni cobertura universal ni calidad de la atención. Por el contrario, entre sus efectos negativos están el incremento de los còstos de atención, la limitación de la cobertura a determinados grupos de población o con determinados servicios (por ejemplo, salud mental y salud oral quedan excluidos), y la aplicación de tecnología y procedimientos médico-quirúrgicos de muy poca efectividad (Terris, 1992).

Los intereses privados obtuvieron importantes concesiones en aspectos donde resultaban afectados: la titularidad de la Seguridad Social, las farmacias privadas continuarían como los únicos proveedores de medicamentos para el primer nivel de atención con el elevado costo que esto representa, escaso control en el consumo de productos químico-farmaceuticos y de tecnología médica, hasta la participación de los centros médicos privados en el sistema público. La concesión de la libre elección de médico incrementó las dificultades de planificación y de realización efectiva de la atención integral a la salud, uno de los principios de la reforma sanitaria. Sin embargo, el elemento central de las negociaciones fue generar mecanismos para asegurar la aportación de fondos públicos que permitieran altos niveles de demanda y consumo de servicios médico-quirúrgicos. En forma adicional se estableció el pago por servicios y el aseguramiento privado como fórmulas de pago para determinados segmentos de población. Al margen de estas negociaciones quedó la derecha política, representada en sus partidos, para favorecer negociaciones directas con los interesados.

Si bien la LGS introdujo algunas regulaciones en la participación de determinados sectores económicos y políticos en el sistema de salud (dirección, gestión, imcompatibilidades, entre otras), el articulado es lo suficientemente ambiguo y permite interpretaciones y negociaciones permanentes que llevan a una alta "flexibilidad" en su implementación.

Otro aspecto que interesa resaltar es la privacidad de las negociaciones políticas que dificulta el control público del poder. Como elemento metodológico, hubo necesidad de acudir al discurso y otras acciones visibles ante esta privacidad (no publicidad y confidencialidad) de las negociaciones-diálogo. La democracia española regula y norma el acceso a la documentación de los debates parlamentarios, cada día más limitados; sin embargo, las negociaciones que se dan "ante" y "post" continúan en la esfera privada. Este hecho es tanto más nocivo, para la democracia, por el "vicio" asentado durante cuarenta años de destrucción y ocultamiento de fuentes documentales. Habría que señalar también como la privacidad de las negociaciones favoreció principalmente a los grupos de interés económico representados en el sistema de salud. Este fenómeno ha sido analizado por Navarro (1989) para explicar las diferentes formas de financiamiento y organización de los servicios de salud, es decir la conformación de Sistema o Servicio Nacional de Salud en otros paises, según el momento de la correlación de fuerzas.

El Gobierno mantuvo en la LGS algunos principios generales referidos a universalización, gratuidad, integración, concepción de salud integral, un sistema sanitario orientado a eliminar las desigualdades económicas y sociales, entre otros, en la primera parte del articulado, lo que le permite conservar una imagen social que le favorece frente a las críticas de derechización, desde algunos sectores del propio PSOE y del sindicato UGT, quienes exigen que se respete el proyecto socialista (Nicolas, 1985).

Finalmente, la Reforma Sanitaria en España "no cierra las puertas a las esperanzas socialistas pero tampoco a otras esperanzas, como las de la derecha que continúa luchando por la privatización-liberalización del Sistema Sanitario" (González, 1985: 55). Con la formulación de la LGS se logra cierta regulación para la conformación del sistema de salud, sin embargo este no es un proceso acabado y establece la posibilidad de continuar diseñando el sistema de acuerdo al momento de las relaciones de fuerza (Navarro, 1989). En $1991 \mathrm{el}$ 
Congreso de Diputados nombró una Comisión, encabezada por el vice-presidente del Banco Central (F. Abril Martorell), destinada a evaluar el Sistema Nacional de Salud y entre sus recomendaciones pidió la privatización del sistema de salud en España, incluyendo tratamiento y servicios preventivos. Ante el informe, se organizó una conferencia nacional de salud de opositores al Informe Abril, que incluía a partidos politicos, federaciones sindicales y organizaciones de salud comunitarias (Terris, 1992). Esto muestra la continuidad del proceso.

\section{RESUMEN}

\section{ERICE, J. E. \& FLORES, R. L. Los Actores} Políticos y Sociales en la Formulación de la Reforma Sanitaria, en España. Cad. Saúde Públ., Rio de Janeiro, 11 (1): 34-44, jan/mar, 1995.

Estudiar la participación de los actores sociales y políticos en el proceso de formulación de la politica de salud, permite comprender las características específicas de organización y funcionamiento de un sistema de salad.

Este trabajo analiza el proceso de formulación de la Ley General de Salud (LGS) en España con el propósito de conocer la relación entre la participación de actores sociales, políticos y económicos en la formulación de la LGS y la conformación del sistema sanitario español.

Para ello, se realizó un estudio de caso durante 1982-1986. Se analizó información documental parlamentaria y prensa médica, principales diarios y revistas e informes y prensa de los actores políticos, sociales y sanitarios.

La primera versión de LGS presentada por el PSOE propuso un sistema de salud con financiamiento y gestión pública para lograr cobertura universal, atención integral, participación comunitaria y educación sanitaria. Esta propuesta fue sometida a compleja negociación con grupos empresariales, sindicatos, profesionales de salud. La LGS aprobada excluye las principios de equidad e incorpora los intereses económicos privados alrededor de la salud: "libre elección" médico-hospitalaria, financiamiento público y la gestión privada del sistema de salud, y establece a la Seguridad Social como rector del sistema.

Palabras Clave: Políticas de Salud; Sistemas de Salud; Actores Sociales; Cobertura en Salud; Seguridad Social

\section{REFERENCIAS BIBLIOGRAFICAS}

CEOE (Confederación Española de Organizaciones Empresariales), 1984. Observaciones al borrador del anteproyecto de ley general de sanidad. Boletín del Consejo General de Colegios Oficiales de Farmacéuticos, Ley General) de Sanidad, S2: 03-05.

CGCOF (Consejo General de Colegios Oficiales de Farmacéuticos), 1984. Información sobre la ley de sanidad. Boletín del Consejo General de Colegios Oficiales de Farmacéuticos, S2: 11-I8.

COMISION REDACTORA-MSC,1984. Anteproyecto de ley general de sanidad. Revista de la Seguridad Social, 22: 163-169.

DEL AGUILA, R. \& MONTORO, R., 1984. El Discurso Político de la Transición Española. Madrid: CIS-Siglo XXI.

DE VICENTE, C., 1985. Entrevista. El Médico, Madrid, 129: 35-38.

FADSP (Fedetación de Asociaciones pats la Defensa de la Sanidad Pública), s/f. Valo-ración del anteproyecto de ley general de sanidad. Boletín de la FADSP, Madrid. (Mimeo.)

FERNANDEZ, J. L., 1986. Entrevista (Delegado Federal de Salud del PSOE). El Médico, Madrid, 183: 51-56.

GARCIA, A., 1987. La transición política en perspectiva. Revista Sistema, 78: 41-46

GOBIERNO ESPAÑOL (CONSEJO DE MINISTROS), 1985. Proyecto de ley general de sanidad. Boletín Official de las Cortes Generales. Congreso de los Diputados, 145-I, 23 abril. (Mimeo.)

GOBIERNO ESPAÑOL (CONGRESO DE LOS DIPUTADOS), 1986. Ley General de Sanidad. Boletín Oficial de las Cortes Generales, Con-greso de los Diputados, 145-V, 09 abril. (Mimeo.)

GONZALEZ, J. L., 1985. Entrevista (Portavoz del Sindicato UGT). El Médico, Madrid, 160: 53-59.

MANCILLA, P. P., 1984. Comentario al anteproyecto de ley general de sanidad. Re-vista de la Seguridad Social, 22: 135-136.

MARAVALL, J. M., 1981. La Política de la Transición, Madrid: Taurus. 
NAVARRO, V., 1989. Why some countries have National Health Insurance, others have National Health Services, and the United States has neither. International Journal of Health Services, 19: 383-404.

NICOLAS, C., 1985. Catorce Borradores en Busca de Ley. El Médico, Madrid, 169: 48-49.

OMC (Organización Médica Colegial), 1985. Dos Años de Relaciones con la Administración. Informes, Propuestas, Documentos Alterna-tivos a los Proyectos Normativos del Gobierno. Madrid: CGCM.
ORTUN, V., 1986. Estructura y política económica de los servicios sanitarios en España. En: Planificación y Economía de la Salud en las Autonomías (Instituto Regional de Estudios de la Comunidad de Madrid), pp. 323-328, Madrid: Consejería de Salud y Bienestar Social.

SANCHEZ, B. M., 1985. Una ley que no gusta a nadie. El Médico, Madrid, 142: 40-45.

TERRIS, M., 1992. Budget cutting and priva-tization: the threat to health. Journal of Public Health Policy, 13: 27-41. 